\title{
Three-Dimensional Imaging of the Lung In Vivo: Work in Progress
}

\author{
Madhav Kavuru, Derek Ney, Elliot K. Fishman, Janet E. Kuhlman, Ralph H. Hruban, \\ and Grover M. Hutchins
}

\begin{abstract}
It has previously been demonstrated that threedimensional (3D) displays of the lung and bronchial tree can be generated from computed tomography (CT) scans of lung specimens. Subsequent refinement of the reconstruction algorithms has allowed highresolution reconstructions of lungs in vivo. With the introduction of low-dose CT scan protocols, use of the technique may become more common in the radiologic community. The many potential clinical applications for 3D imaging of the lung include an aid to bronchoscopy and improved surgical planning. We present the technical details for 3D imaging of the lungs in vivo as well as three representative case studies.
\end{abstract}

Copyright $\odot 1991$ by W.B. Saunders Company

KEY WORDS: three-dimensional imaging, image processing, lung parenchyma.

$\mathbf{T}$ THREE-DIMENSIONAL (3D) reconstruction of computed tomographic (CT) data has been used in various clinical applications, particularly in displaying the bony skeleton, and more recently soft tissue and muscular structures. ${ }^{1-5}$ Originally we implemented a novel reconstruction algorithm, the volumetric rendering technique, to create highly accurate $3 \mathrm{D}$ images of musculoskeletal pathology and anatomy. These algorithms were subsequently modified to generate high-resolution 3D images of lung parenchyma and significant portions of the bronchial tree from lung specimens. ${ }^{6}$ Similar image processing techniques were then applied to the in vivo lung. To optimize the technique and decrease user interaction we developed a volume filling technique that automatically identifies the lung and removes all surrounding unwanted tissue. The details of this study provide the basis for this article.

\section{MATERIALS AND METHODS}

Patients were scanned on a third generation CT scanner (Siemens DR-H, Siemens Medical Systems, Iselin, NJ) using a standard protocol of $4 \mathrm{~mm}$ slice thickness, $3 \mathrm{~mm}$ slice interval (cases 1 and 2) or $8 \mathrm{~mm} \times 8 \mathrm{~mm}$ (case 3), 3 seconds, $230 \mathrm{mAs}$, and $125 \mathrm{KVp}$. The volume of interest only was scanned, generating approximately 35 scans. Because of the importance of reducing breathing artifacts, the patients were instructed to lie still and breathe shallowly. We have found that this minimizes motion artifacts. Scan time was approximately 6 to 8 minutes.

After scanning, the image data was transferred to a free-standing imaging system via 9-track magnetic tape. The transfer process takes less than 5 minutes. The imaging system consists of a Pixar Image Computer (PIXAR, San Rafael, CA) with a host Sun workstation (Sun Microsystems, Mountain View, CA). All 3D reconstructions were done using the Doctor software package (Johns Hopkins Medical Institutions, Baltimore, MD).

To properly image the lung and overcome partial volume effects a new size classification algorithm was introduced in our previous study of lung specimens. It was demonstrated that the qualitative features of the lung parenchyma could still be visualized while displaying the major components of the bronchial tree. A problem unique to the lung in vivo is the presence of surrounding soft tissue and bone structures of equal or higher density. This tissue must be identified and removed in order that it not obscure the lung tissue when imaged. For this purpose a two-part lung extraction program was introduced. First the lungs are automatically located and filled in based on their Hounsfield value. Curves are then extracted from the newly formed edges. At this point one can edit the curves (eg, remove one lung) or allow the algorithm to proceed with the $3 \mathrm{D}$ reconstruction.

The lung isolation technique begins by finding a point located centrally in one of the lungs. The point is found by starting at the center of the volume scanned and searching radially until a volume element (voxel) with low attenuation is found. As long as the patient was approximately centered in the scan field, the voxel will represent lung. This initial voxel is the seed point for a volume filling algorithm. The algorithm then looks for voxels neighboring the seed voxel that have low attenuation (see Fig 1). Each of these voxels is then treated as a seed voxel and the process is repeated. Using this method, the entire volume of the lung is identified. The resulting volume mask encompasses the lung, the lung pleura, and a small amount of surrounding muscle tissue. Because the pleura and muscle tissue are of slightly higher density, it is necessary to remove these voxels in order to clearly visualize the inner structures of the lung. An erosion technique is used to remove the outer 2 voxels from the lung volume determined with the volume filling technique. The erosion process consists of removing any voxel

From the Department of Radiology, The Johns Hopkins Medical Institutions, Baltimore, $M D$.

Supported in part by a grant from OxyChem Corporation.

Presented at Radiological Society of North America '89.

Address reprint requests to Derek Ney, Department of Radiology, The Johns Hopkins Medical Institutions, $600 \mathrm{~N}$ Wolfe St, Baltimore, MD 21205.

Copyright $\odot 1991$ by W.B. Saunders Company

0897-1889/91/0403-0001\$03.00/0 


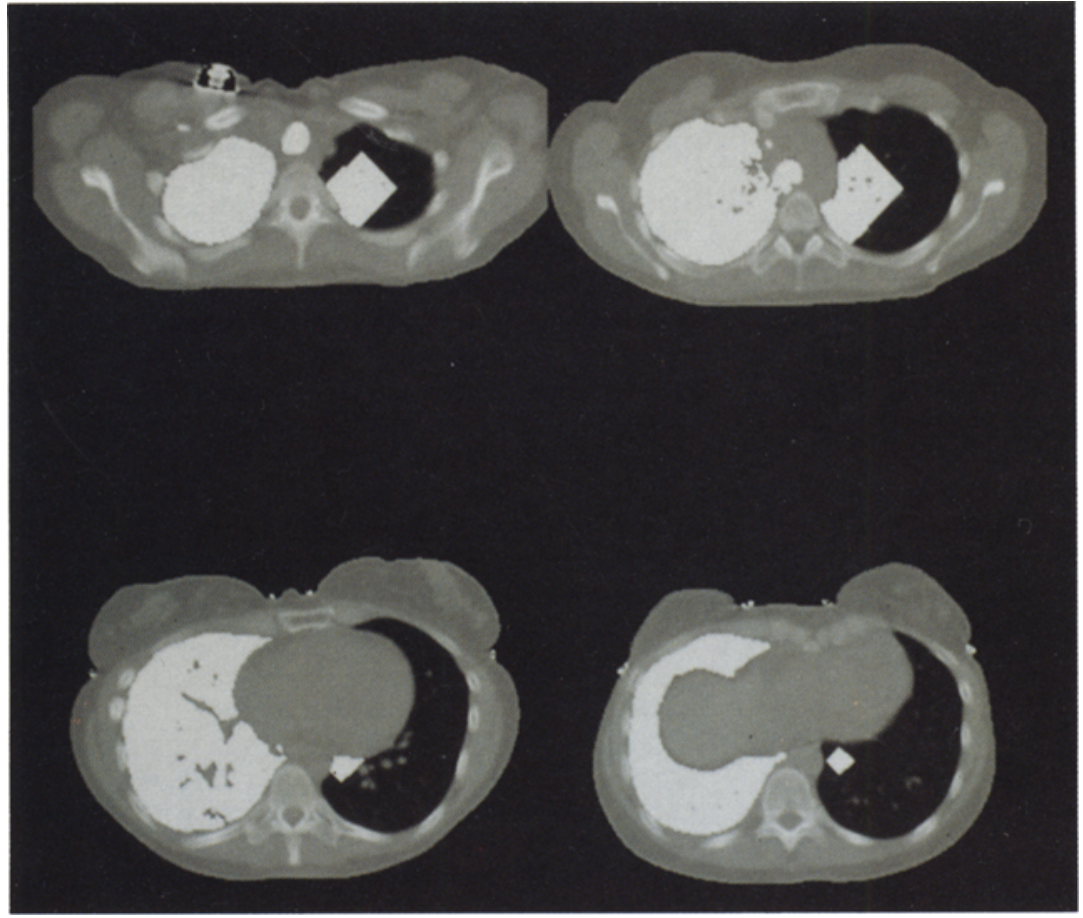

Fig 1. Successive images of the volume fill. The bright white areas represent areas identified as lung. The algorithm iteratively expands these areas until the whole lung has been filled. that is within 2 voxels of the edge of the lung volume itself. This is implemented by smoothing the volume masks and then thresholding the masks to remove the outermost members of the mask. We experimented with the amount of tissue erosion and determined that 2 voxels produced optimal images. The last step in the lung isolation technique is to extract contours from the volume mask (Fig 2).

The contours extracted for the lung volume may in some cases need to be edited by the user to more accurately encompass the entire volume to be imaged. This process takes less than 5 minutes and is only necessary in cases in which lesions are present on or near the pleura.

The contours are then used to select the volume elements that are to be imaged in the imaging process. This portion of the volume is rendered using the Volumetric Rendering Technique. This process is explained in detail in references 6,7 , and 8 . The rendering process produces 72 images with viewing orientation spaced around the spinal or pelvic axis.

Fig 2. Curves defining the edges of the lung and trachea. 
Fig 3. Three-dimensional images of the lung reconstructed from $\mathrm{CT}$. The lungs are normal in this case. Notice the clear visualization of the bronchial tree.

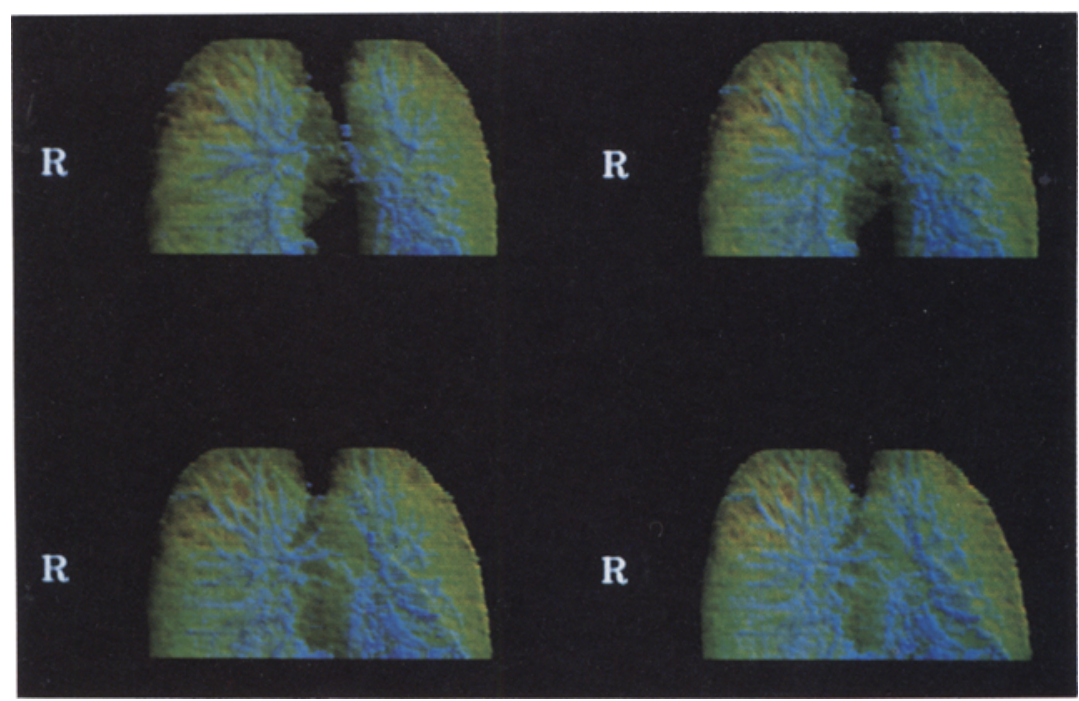

Case 1

This 48-year-old woman presented with a history of left chest pain that was felt to be musculoskeletal in origin. The patient had similar episodes over the previous dozen years although no source was found. A bone scan was done and suggested some increased activity in the region of the left third rib. A CT was then ordered for further definition of this region. The study was done with consecutive $4 \mathrm{~mm}$ thick sections at $3 \mathrm{~mm}$ intervals through the axilla and brachial plexus region. Three-dimensional reconstruction of the lungs showed a well defined bronchial tree and normal lung paren-

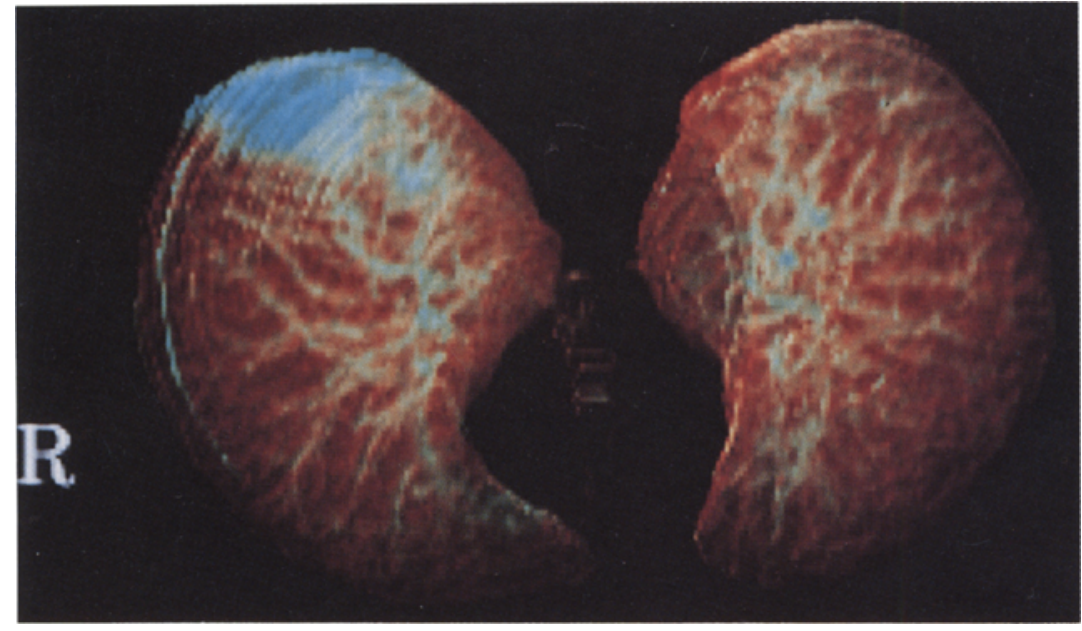

Fig 4. Three-dimensional images of the lung displaying a pulmonary infarct in the right upper lung (case 2). 


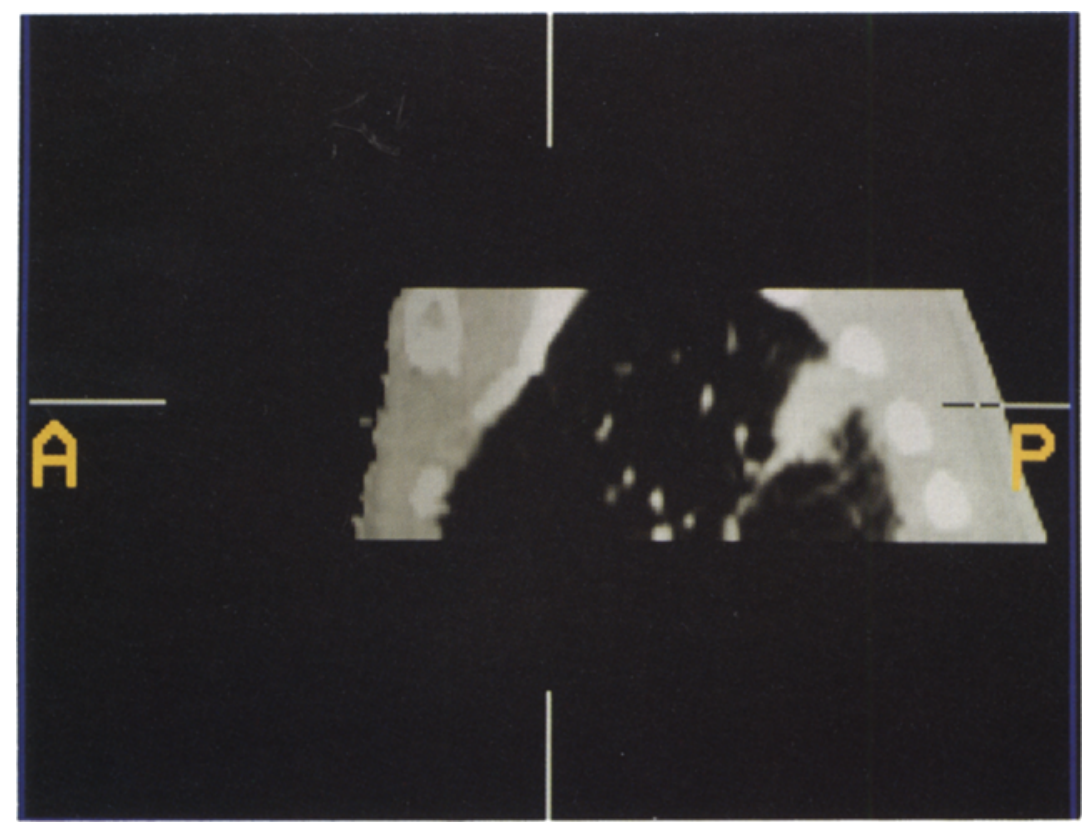

Fig 5. Multiplanar reformatting done with Orthotool shows the feeding vessel responsible for the pulmonary infarct (arrows) (case 2). chyma, as in Fig 3. No evidence of soft tissue or bony mass was seen. The patient's symptoms resolved on nonsteroidal anti-inflammatory medication.

\section{Case 2}

This 29-year-old man was admitted for fever, chills, and shortness of breath. Anterior upper chest pain was also noted for deep breaths and movement. CT of the lungs showed a pleural- based lesion in the right upper lung compatible with septic emboli. Bilateral pleural effusions were also seen. Three-dimensional reconstruction of the lungs confirmed the presence of a pulmonary infarct in the right upper lung. The infarct is clearly seen in Fig 4. The reconstructed lung was analyzed further by using Orthotool $^{9}$ (Johns Hopkins Medical Institutions, Baltimore, MD) to find the feeding vessel responsible for the infarct (Fig 5). As can be
Fig 6. Three-dimensional images of lung nodules resulting from metastatic osteogenic sarcoma (case 3).

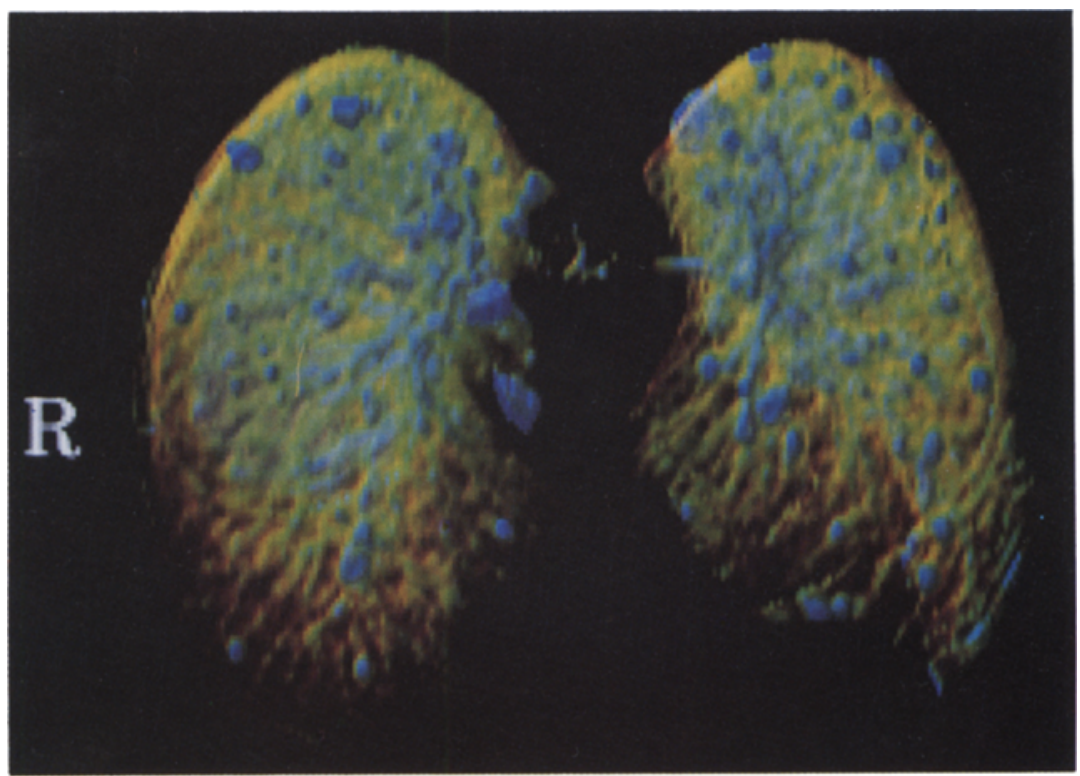


seen there is excellent agreement between the findings in two and three dimensional CT.

\section{Case 3}

The patient was a 62-year-old man who presented approximately 2 months prior to admission with diplopia and prominence of the right temporal region. CT scans of the head demonstrated a large blastic lesion in the right sphenoid bone and clivus. A bone scan revealed multiple areas of increased tracer accumulation. A CT of the chest and abdomen was done and multiple lung metastases as well as multiple blastic bone lesions were detected. A percutaneous right iliac crest bone biopsy was done and was consistent with osteogenic sarcoma. The lung lesions ranged in size from 5 to $18 \mathrm{~mm}$ and several of them were of high CT attenuation compatible with metastatic osteogenic sarcoma. A $3 \mathrm{D}$ reconstruction of the lung was used for surgical planning if lung resection was contemplated. The 3D reconstruction seen in Fig 6 reveals the extent of the pulmonary metastases. Most of the bronchial tree was edited out to improve the transparency of the lungs and emphasize the parenchyma where the nodules are quite striking. Due to the extent of the bone lesions resection of the lung nodules was not done and the patient was referred for chemotherapy.

\section{DISCUSSION}

Three-dimensional imaging in radiology has begun to evolve into a valuable imaging study for several specific clinical problems. This is particularly true for complex orthopedic cases and craniofacial surgery planning. With the introduction of improved reconstruction algorithms and new, second and subsecond scanners, data sets for 3D imaging can be rapidly collected and processed. It is the application of this technology to other clinical problems that holds great potential for improved clinical decision making.

Conventional transaxial computed tomography has long been the modality of choice for evaluating lung pathology due to its superior contrast resolution and cross-sectional imaging. Three-dimensional imaging of the lung takes advantage of the excellent contrast resolution to generate highly informative displays of the lung anatomy. Moreover, multiplanar reformatting allows one to focus on the planes of interest.

It was previously concluded that one can indeed display a number of disease entities present in lung specimens. When axial CT reconstructions were compared with corresponding pathological specimens, accurate, one-toone, radiologic-pathologic correlations were demonstrated for extensive pulmonary emphysema and multiple pulmonary infarcts. ${ }^{6}$

The recent introduction of low-dose CT scan protocols for the lung suggests that the radiation dose associated with $3 \mathrm{D}$ reconstruction can be decreased significantly. ${ }^{10}$ The rationale behind this is that the higher contrast within the lung compared to solid organs makes the detection of pathologic changes less dependent on image noise. Other things being equal, radiation dose is directly proportional to the milliamperesecond value. It is conceivable that the total radiation dose can be lowered by an order of magnitude or more and still generate highresolution reconstructions of the lung by scanning at proportionately lower milliampere settings. In situations where the bronchial tree is the primary subject of interest this technique is all the more feasible since the contrast at the air-bronchus interface is greater than the contrast in the rest of the lung parenchyma.

Initial concerns about patient respiratory motion have been handled adequately by scanning technique so that displays of the tracheobronchial tree are routinely achieved with shallow breathing. One can expect motion artifact to become even less of a problem using the new subsecond scanners or helical scanning. Helical scanning promises to be the future study of choice for 3D imaging of the lung. Helical scanning would allow the acquisition of a volume of the lung in one breathhold. This would allow for images that would exhibit no motion artifact even in the parts of the lung near the diaphragm. ${ }^{11}$

It has previously been demonstrated that injection of contrast into the pulmonary arteries of lung specimens allows detailed reconstruction of the pulmonary vascular tree. The use of constrast in vivo should generate comparable reconstructions. Three-dimensional imaging of the tracheobronchial tree in conjunction with the pulmonary vascular tree provides a great 
deal of functional information about the lung. In the cases where contrast is undesirable magnetic resonance angiography could provide the complementary information. Other useful information that can be gained from 3D imaging of the lungs is very precise calculation of lung volume. The algorithm used in our studies automatically extracts the lungs from the CT slices and generates a set of curves defining the lung border. The area within these curves can easily be integrated and the total lung volume calculated.

The generation of 3D models of the lungs and bronchial tree has many potential clinical applications including: (1) a true anatomic guide for bronchoscopy, particularly in complex cases; (2) more precise planning of surgical resections in patients with primary lung tumors or multiple lung metastases; (3) incorporation into 3D radiation therapy treatment planning; (4) tumor volumetrics; and (5) medical student and resident teaching of lung anatomy and pathology.
At present we have several goals that should help define the true utility of this technique. A systematic study of resolution at different radiation dosages as measured by degree of branching of bronchi and vessels and detection rate for various lesions would allow us to optimize the scan parameters for comparable resolution at a lower dose. By implementing the new protocols we hope to further improve the quality of the reconstructions of the tracheobronchial tree to the point where it has practical utility in bronchoscopy. Finally as an aid for planning of surgical resections and oncologic therapy we are working to develop new algorithms to measure tumor volumes.

New low dose CT scanning protocols make these techniques a viable option for use in the daily clinical practice. The several potential clinical applications of 3D imaging of the lung in vivo warrants further investigation.

\section{ACKNOWLEDGMENT}

Special thanks to Robert Gilligan, MD and Jay Baker.

\section{REFERENCES}

1. Fishman EK, Drebin RA, Magid D, et al: Volumetric rendering techniques: Applications for three-dimensional imaging of the hip. Radiology 163:737-738, 1987

2. Fishman EK, Magid D, Ney DR, et al: Threedimensional imaging and display of musculoskeletal anatomy. J Comput Assist Tomogr 12(3):465-469, 1988.

3. Kuhlman JE, Fishman EK, Ney DR, Magid D: Complex shoulder trauma: Three dimensional CT imaging. Orthopedics 11(2):1561-1563, 1988

4. Fishman EK, Ney DR, Magid D, et al: Three dimensional imaging of the vascular tree. Dyn Cardio Imag 2(1):55-60, 1989

5. Stern RL, Cline HE, Johnson GA, et al: Threedimensional imaging of the thoracic cavity. Invest Radiol 24:282-288, 1989

6. Ney DR, Kuhlman JE, Hruban RH, et al: Threedimensional CT-Volumetric Reconstruction and display of the bronchial tree. Invest Radiol 25(6):736-742, 1990

7. Drebin RA, Carpenter L, Hanrahan P: Volume Rendering. Computer Graphics. Special Interest Group Graphics '88 Proceedings 22(4):65-74, 1988

8. Ney DR, Drebin RA, Fishman EK, et al: Voumetric rendering of computed tomography data: Principles and techniques. Computer Graphics App 10(2):24-32, 1990

9. Ney DR, Drebin RA, Fishman EK, et al: Interactive real-time multiplanar CT imaging. Radiology 170:275-276, 1989

10. Naidich DP, Marshall CH, Gribbin C, et al: Low-dose CT of the lungs: Preliminary observations. Radiology 175 : 729-731, 1990

11. Vock P, Soucek M, Daepp M, et al: Lung: Spiral volumetric CT with single-breath-hold technique. Radiology 176:864-867, 1990 